\title{
BMC Ophthalmology reviewer acknowledgement 2014
}

Catherine M Rice ${ }^{*}$ and Diana M Marshall

\section{Contributing reviewers}

The editors of BMC Ophthalmology would like to thank all our reviewers who have contributed to the journal in Volume 14 (2014).

Osama Ababneh
Jordan

Mohammed Abdull

UK

Marwan Abouammoh

Saudi Arabia

Grazyna Adamus

USA

Aniruddha Agarwal

USA

Ashish Ahuja

USA

Tan Aik Kah

Malaysia

Hideo Akiyama

Japan

Luciana Alencar

Brazil

Christiane Al-Haddad

Lebanon

Aliaa Ali

Egypt

Mohammad Ali

India

Majed Alkharashi

Saudi Arabia
Fowzan Alkuraya
Saudi Arabia

Riham Allam

Egypt

Winfried Amoaku

UK

\section{Marcus Ang}

Singapore

Nicola Anstice

New Zealand

Florent Aptel

France

Ilir Arapi

Italy

Samuel Arba Mosquera

Spain

Luis Arias

Spain

Paul Artes

Canada

Maria Angeles Asencio

Spain

Salman Asghar

Pakistan

Ioannis Aslanis Aslanides

Greece
Sam Ebenezer Athikarisamy

Australia

Francesco Attanasio

Italy

Venkata Avadhanam

UK

Abdulkabir Ayanniyi

Nigeria

Ramesh Ayyala

USA

Simon Bababeygy

USA

Simon Backhouse

Australia

Sehyun Baek

Korea, South

Berker Bakbak

Turkey

Konstantinos Balaskas

UK

Mehmet Balci

Turkey

Melike Balikoglu-Yilmaz

Turkey

Touka Banaee

Iran

* Correspondence: Catherine.Rice@biomedcentral.com

BioMed Central, Floor 6, 236 Gray's Inn Road, London WC1X 8HB, UK

\section{() Biomed Central}

(c) 2015 Rice and Marshall; licensee BioMed Central. This is an Open Access article distributed under the terms of the Creative Commons Attribution License (http://creativecommons.org/licenses/by/4.0), which permits unrestricted use, distribution, and reproduction in any medium, provided the original work is properly credited. The Creative Commons Public Domain Dedication waiver (http://creativecommons.org/publicdomain/zero/1.0/) applies to the data made available in this article, unless otherwise stated. 


\begin{tabular}{|c|c|c|}
\hline $\begin{array}{l}\text { Katayoon Baradaran Ebrahimi } \\
\text { USA }\end{array}$ & $\begin{array}{l}\text { Jill Carlton } \\
\text { UK }\end{array}$ & $\begin{array}{l}\text { Kam-Lung, Kelvin Chong } \\
\text { Hong Kong }\end{array}$ \\
\hline $\begin{array}{l}\text { Piero Barboni } \\
\text { Italy }\end{array}$ & $\begin{array}{l}\text { Zia Carrim } \\
\text { UK }\end{array}$ & $\begin{array}{l}\text { Sung Kun Chung } \\
\text { Korea, South }\end{array}$ \\
\hline $\begin{array}{l}\text { John Barbur } \\
\text { UK }\end{array}$ & $\begin{array}{l}\text { Marissa Carter } \\
\text { USA }\end{array}$ & $\begin{array}{l}\text { Nerida Cole } \\
\text { Australia }\end{array}$ \\
\hline $\begin{array}{l}\text { Brendan Barrett } \\
\text { UK }\end{array}$ & $\begin{array}{l}\text { Robert Casson } \\
\text { Australia }\end{array}$ & $\begin{array}{l}\text { Miriam Conway } \\
\text { UK }\end{array}$ \\
\hline $\begin{array}{l}\text { John Barry } \\
\text { UK }\end{array}$ & $\begin{array}{l}\text { Ingele Casteels } \\
\text { Belgium }\end{array}$ & $\begin{array}{l}\text { Jessica Cooke Bailey } \\
\text { USA }\end{array}$ \\
\hline $\begin{array}{l}\text { Peter Barry } \\
\text { Ireland }\end{array}$ & $\begin{array}{l}\text { Heidi Cate } \\
\text { UK }\end{array}$ & $\begin{array}{l}\text { Ciro Costagliola } \\
\text { Italy }\end{array}$ \\
\hline $\begin{array}{l}\text { Robert Barry } \\
\text { UK }\end{array}$ & $\begin{array}{l}\text { Cestmir Cejka } \\
\text { Czech Republic }\end{array}$ & $\begin{array}{l}\text { John Crews } \\
\text { USA }\end{array}$ \\
\hline $\begin{array}{l}\text { Ken Bassett } \\
\text { Canada }\end{array}$ & $\begin{array}{l}\text { Mario Chacon } \\
\text { Mexico }\end{array}$ & $\begin{array}{l}\text { Valente Cristiana } \\
\text { Italy }\end{array}$ \\
\hline $\begin{array}{l}\text { Roberto Bellucci } \\
\text { Italy }\end{array}$ & $\begin{array}{l}\text { Clara Chan } \\
\text { Canada }\end{array}$ & $\begin{array}{l}\text { Phillippa Cumberland } \\
\text { UK }\end{array}$ \\
\hline $\begin{array}{l}\text { Antonio Benito } \\
\text { Spain }\end{array}$ & $\begin{array}{l}\text { Errol Chan } \\
\text { Singapore }\end{array}$ & $\begin{array}{l}\text { Mary Daly } \\
\text { USA }\end{array}$ \\
\hline $\begin{array}{l}\text { Kanchan Bhan } \\
\text { UK }\end{array}$ & $\begin{array}{l}\text { William Charman } \\
\text { UK }\end{array}$ & $\begin{array}{l}\text { Erika Damato } \\
\text { UK }\end{array}$ \\
\hline $\begin{array}{l}\text { Alexandre Bisdorff } \\
\text { Luxembourg }\end{array}$ & $\begin{array}{l}\text { Tanveer Chaudhry } \\
\text { Pakistan }\end{array}$ & $\begin{array}{l}\text { Vivek Dave } \\
\text { India }\end{array}$ \\
\hline $\begin{array}{l}\text { Paul Bishop } \\
\text { UK }\end{array}$ & $\begin{array}{l}\text { Sunita Chaurasia } \\
\text { India }\end{array}$ & $\begin{array}{l}\text { Annelies De Klein } \\
\text { Netherlands }\end{array}$ \\
\hline $\begin{array}{l}\text { Michael Boland } \\
\text { USA }\end{array}$ & $\begin{array}{l}\text { Angela Chen } \\
\text { USA }\end{array}$ & $\begin{array}{l}\text { Gabor Deak } \\
\text { Austria }\end{array}$ \\
\hline $\begin{array}{l}\text { Beatrice Bourghardt Peebo } \\
\text { Sweden }\end{array}$ & $\begin{array}{l}\text { Ching-Shih Chen } \\
\text { USA }\end{array}$ & $\begin{array}{l}\text { Dawn Decarlo } \\
\text { USA }\end{array}$ \\
\hline $\begin{array}{l}\text { Mete Bozkurt } \\
\text { Turkey }\end{array}$ & $\begin{array}{l}\text { Katherine Chen } \\
\text { USA }\end{array}$ & $\begin{array}{l}\text { Alastair Denniston } \\
\text { UK }\end{array}$ \\
\hline $\begin{array}{l}\text { Beau Bruce } \\
\text { USA }\end{array}$ & $\begin{array}{l}\text { Yihe Chen } \\
\text { USA }\end{array}$ & $\begin{array}{l}\text { Alexandre Denoyer } \\
\text { France }\end{array}$ \\
\hline $\begin{array}{l}\text { Paolo Brusini } \\
\text { Italy }\end{array}$ & $\begin{array}{l}\text { Clarissa Cheng } \\
\text { Singapore }\end{array}$ & $\begin{array}{l}\text { Manishi Desai } \\
\text { USA }\end{array}$ \\
\hline $\begin{array}{l}\text { Franziska Bucher } \\
\text { Germany }\end{array}$ & $\begin{array}{l}\text { Andreea Chih } \\
\text { USA }\end{array}$ & $\begin{array}{l}\text { Vasilios Diakonis } \\
\text { Greece }\end{array}$ \\
\hline $\begin{array}{l}\text { Ibrahim Buttanri } \\
\text { Turkey }\end{array}$ & $\begin{array}{l}\text { Pauline Cho Hong } \\
\text { Kong }\end{array}$ & $\begin{array}{l}\text { Andrew Dick } \\
\text { UK }\end{array}$ \\
\hline $\begin{array}{l}\text { Halil Çagatay } \\
\text { Turkey }\end{array}$ & $\begin{array}{l}\text { Hyuk Jin Choi } \\
\text { Korea, South }\end{array}$ & $\begin{array}{l}\text { Xiaoyan Ding } \\
\text { China }\end{array}$ \\
\hline $\begin{array}{l}\text { Carlo Cagini } \\
\text { Italy }\end{array}$ & $\begin{array}{l}\text { Kup-Sze Choi } \\
\text { Hong Kong }\end{array}$ & $\begin{array}{l}\text { Ali Djalilian } \\
\text { USA }\end{array}$ \\
\hline
\end{tabular}




\begin{tabular}{|c|c|c|}
\hline $\begin{array}{l}\text { Mangat Ram Dogra } \\
\text { India }\end{array}$ & $\begin{array}{l}\text { Irene Floriani } \\
\text { Italy }\end{array}$ & $\begin{array}{l}\text { Jonathan Gibson } \\
\text { UK }\end{array}$ \\
\hline $\begin{array}{l}\text { Thomas Dohlman } \\
\text { USA }\end{array}$ & $\begin{array}{l}\text { Paolo Fogagnolo } \\
\text { Italy }\end{array}$ & $\begin{array}{l}\text { Fleur Goezinne } \\
\text { Netherlands }\end{array}$ \\
\hline $\begin{array}{l}\text { Kimberly Drenser } \\
\text { USA }\end{array}$ & $\begin{array}{l}\text { Angie Fong } \\
\text { Hong Kong }\end{array}$ & $\begin{array}{l}\text { E-Shawn Goh } \\
\text { Singapore }\end{array}$ \\
\hline $\begin{array}{l}\text { Chiara Eandi } \\
\text { Italy }\end{array}$ & $\begin{array}{l}\text { Alex Fonollosa Calduch } \\
\text { Spain }\end{array}$ & $\begin{array}{l}\text { Gokcen Gokce } \\
\text { Turkey }\end{array}$ \\
\hline $\begin{array}{l}\text { Deepak Edward } \\
\text { Saudi Arabia }\end{array}$ & $\begin{array}{l}\text { Gary Foster } \\
\text { USA }\end{array}$ & $\begin{array}{l}\text { Dan Gold } \\
\text { USA }\end{array}$ \\
\hline $\begin{array}{l}\text { Nathan Efron } \\
\text { Australia }\end{array}$ & $\begin{array}{l}\text { Nikitas Fountoulakis } \\
\text { Greece }\end{array}$ & $\begin{array}{l}\text { David Goldblum } \\
\text { Switzerland }\end{array}$ \\
\hline $\begin{array}{l}\text { Allen Eghrari } \\
\text { USA }\end{array}$ & $\begin{array}{l}\text { Ian Francis } \\
\text { Australia }\end{array}$ & $\begin{array}{l}\text { Johannes Gonnermann } \\
\text { Germany }\end{array}$ \\
\hline $\begin{array}{l}\text { Alvin Eisner } \\
\text { USA }\end{array}$ & $\begin{array}{l}\text { Sandra Franco } \\
\text { Portugal }\end{array}$ & $\begin{array}{l}\text { J Gonzalez-Meijome } \\
\text { Portugal }\end{array}$ \\
\hline $\begin{array}{l}\text { Tom Eke } \\
\text { UK }\end{array}$ & $\begin{array}{l}\text { Jeffrey Freedman } \\
\text { USA }\end{array}$ & $\begin{array}{l}\text { Günther Grabner } \\
\text { Austria }\end{array}$ \\
\hline $\begin{array}{l}\text { Bengu Ekinci Koktekir } \\
\text { Turkey }\end{array}$ & $\begin{array}{l}\text { Amanda French } \\
\text { Australia }\end{array}$ & $\begin{array}{l}\text { Enrique Graue-Hernández } \\
\text { Mexico }\end{array}$ \\
\hline $\begin{array}{l}\text { Mohamed El Sanharawi } \\
\text { France }\end{array}$ & $\begin{array}{l}\text { Paolo Frezzotti } \\
\text { Italy }\end{array}$ & $\begin{array}{l}\text { Philip Griffiths } \\
\text { Gibraltar }\end{array}$ \\
\hline $\begin{array}{l}\text { Yamine El Sayed } \\
\text { Egypt }\end{array}$ & $\begin{array}{l}\text { Fusako Fusimura } \\
\text { Japan }\end{array}$ & $\begin{array}{l}\text { Inna Grishkan } \\
\text { USA }\end{array}$ \\
\hline $\begin{array}{l}\text { Mostafa Elgohary } \\
\text { UK }\end{array}$ & $\begin{array}{l}\text { Jeremias Galletti } \\
\text { Argentina }\end{array}$ & $\begin{array}{l}\text { Andrzej Grzybowski } \\
\text { Poland }\end{array}$ \\
\hline $\begin{array}{l}\text { Sitki Ermis } \\
\text { Turkey }\end{array}$ & $\begin{array}{l}\text { Juan Eduardo Gallo } \\
\text { Argentina }\end{array}$ & $\begin{array}{l}\text { Laurent Guilloton } \\
\text { France }\end{array}$ \\
\hline $\begin{array}{l}\text { Marie-Helene Errera } \\
\text { France }\end{array}$ & $\begin{array}{l}\text { Alfredo Garcia-Layana } \\
\text { Spain }\end{array}$ & $\begin{array}{l}\text { Priya Gupta } \\
\text { Canada }\end{array}$ \\
\hline $\begin{array}{l}\text { Richard Fairless } \\
\text { Germany }\end{array}$ & $\begin{array}{l}\text { Sunir Garg } \\
\text { USA }\end{array}$ & $\begin{array}{l}\text { Nigel Hall } \\
\text { UK }\end{array}$ \\
\hline $\begin{array}{l}\text { Miguel Faria-Ribeiro } \\
\text { Portugal }\end{array}$ & $\begin{array}{l}\text { Zisis Gatzioufas } \\
\text { Switzerland }\end{array}$ & $\begin{array}{l}\text { Inderraj Hanspal } \\
\text { UK }\end{array}$ \\
\hline $\begin{array}{l}\text { Sepehr Feizi } \\
\text { Iran }\end{array}$ & $\begin{array}{l}\text { Jian Ge } \\
\text { China }\end{array}$ & $\begin{array}{l}\text { Hideaki Hara } \\
\text { Japan }\end{array}$ \\
\hline $\begin{array}{l}\text { Yifan Feng } \\
\text { China }\end{array}$ & $\begin{array}{l}\text { Ronald Gentile } \\
\text { USA }\end{array}$ & $\begin{array}{l}\text { Takeshi Harayama } \\
\text { Japan }\end{array}$ \\
\hline $\begin{array}{l}\text { Maryam Ferdousi } \\
\text { UK }\end{array}$ & $\begin{array}{l}\text { Ilias Georgalas } \\
\text { Greece }\end{array}$ & $\begin{array}{l}\text { Sveinn Hakon Hardarson } \\
\text { Iceland }\end{array}$ \\
\hline $\begin{array}{l}\text { Roberto Fernandez Buenaga } \\
\text { Spain }\end{array}$ & $\begin{array}{l}\text { Joann Giaconi } \\
\text { USA }\end{array}$ & $\begin{array}{l}\text { Andrew Harrison } \\
\text { USA }\end{array}$ \\
\hline $\begin{array}{l}\text { Antonio Ferreras } \\
\text { Spain }\end{array}$ & $\begin{array}{l}\text { Andrea Giani } \\
\text { Italy }\end{array}$ & $\begin{array}{l}\text { Noriyasu Hashida } \\
\text { Japan }\end{array}$ \\
\hline
\end{tabular}

Mangat Ram Dogra
India

Thomas Dohlman

USA

Kimberly Drenser

Chiara Eandi

Deepak Edward

Saudi Arabia

Nathan Efron

Allen Eghrari

Alvin Eisner

USA

Tom Eke

Bengu Ekinci Koktekir

Mohamed El Sanharawi

France

Yamine El Sayed

ypt

Mostafa Elgohary

Sitki Ermis

Turkey

Marie-Helene Errera

Richard Fairless

Miguel Faria-Ribeiro

Portuga

Sepehr Feizi

Yifan Feng

Maryam Ferdousi

Roberto Fernandez Buenaga

Spain

Spain
Irene Floriani

Paolo Fogagnolo

Italy

Angie Fong

Alex Fonollosa Calduch

USA

Nikitas Fountoulakis

Ian Francis

Sandra Franco

Portugal

Jeffrey Freedman

Amanda French

Paolo Frezzotti

Italy

usako Fusimura

Jeremias Galletti

Juan Eduardo Gallo

Argentina

Alfredo Garcia-Layana

Sunir Garg

Zisis Gatzioufas

Switzerland

ian Ge

Ronald Gentile

Ilias Georgalas

Joann Giaconi

Italy
Jonathan Gibson

Fleur Goezinne

etherlands

E-Shawn Goh

Gokcen Gokce

Dan Gold

USA

David Goldblum

witzerland

Johannes Gonnermann

J Gonzalez-Meijome

Portuga

Günther Grabner

Austria

Enrique Graue-Hernández

Philip Griffiths

Gibraltar

Inna Grishkan

Andrzej Grzybowski

France

Priya Gupta

Nigel Hall

Inderraj Hanspal

UK

ki Hara

Takeshi Harayama

Iceland

Andrew Harrison

Noriyasu Hashida 


\begin{tabular}{|c|c|c|}
\hline Scott Hau & Makoto Inoue & Kishore Reddy Katikireddy \\
\hline UK & Japan & USA \\
\hline Miao He & Jenny Ip & Andreas Katsanos \\
\hline China & Australia & Greece \\
\hline Jiucheng He & Murat Irkec & Sushmita Kaushik \\
\hline USA & Turkmenistan & India \\
\hline Shikun He & Fakir M Amirul Islam & Srinivasan Kavitha \\
\hline USA & Australia & India \\
\hline Owen Hee & Domagoj Ivastinovic & Ryo Kawasaki \\
\hline Singapore & Austria & Japan \\
\hline Steffen Heegaard & Nieraj Jain & Stephen Kaye \\
\hline Denmark & USA & UK \\
\hline James Hejtmancik & Tamarra James-Todd & Ramesh Kekunnaya \\
\hline USA & USA & India \\
\hline Taichi Hikichi & Vikram Jayaram & Takeshi Kezuka \\
\hline Japan & USA & Japan \\
\hline Cornelia Hirn & Vishal Jhanji & Jyoti Khadka \\
\hline Switzerland & Hong Kong & Australia \\
\hline Juha Holopainen & Jian Ji & Rohit Khanna \\
\hline Finland & China & India \\
\hline Milton Hom & Animesh Jindal & Ahmad Kheirkhah \\
\hline USA & India & USA \\
\hline Shigeru Honda & Björn Johansson & Mee Kum Kim \\
\hline Japan & Sweden & Korea, South \\
\hline Sadao Hori & Choun-Ki Joo & Tae-Im Kim \\
\hline Japan & Korea, South & Korea, South \\
\hline Huang Houbin & Jong Hwa Jun & Anthony King \\
\hline China & Korea, South & UK \\
\hline Paul Houghtaling & Rim Kahloun & James Kirwan \\
\hline USA & Tunisia & UK \\
\hline Xiufang Hu & Freny Kalapesi & Ronald Klein \\
\hline China & Australia & USA \\
\hline Ruth Huna-Baron & Yogita Kanan & Victoria Knudsen \\
\hline Israel & USA & USA \\
\hline Rahat Husain & Costas Karabatsas & Gergana Kodjebacheva \\
\hline Singapore & Greece & USA \\
\hline Jeong-Min Hwang & Murat Karacorlu & Nageswara Rao Kollu \\
\hline Korea, South & Turkey & USA \\
\hline Takeshi Ide & Dimitrios Karagiannis & Georgios Kontadakis \\
\hline USA & Greece & Greece \\
\hline Michele Iester & Newton Kara-Junior & Juergen Kopitz \\
\hline Italy & Brazil & Germany \\
\hline Yasushi Ikuno & Marzieh Katibeh & Gabor Koranyi \\
\hline Japan & Iran & Sweden \\
\hline
\end{tabular}




\begin{tabular}{|c|c|c|}
\hline $\begin{array}{l}\text { Bobby Korn } \\
\text { USA }\end{array}$ & $\begin{array}{l}\text { Chen Le } \\
\text { China }\end{array}$ & $\begin{array}{l}\text { Ling Luo } \\
\text { China }\end{array}$ \\
\hline $\begin{array}{l}\text { Suheyla Kose } \\
\text { Turkey }\end{array}$ & $\begin{array}{l}\text { Hyun Soo Lee } \\
\text { Korea, South }\end{array}$ & $\begin{array}{l}\text { Douglas Lyall } \\
\text { UK }\end{array}$ \\
\hline $\begin{array}{l}\text { Parvaiz Koul } \\
\text { India }\end{array}$ & $\begin{array}{l}\text { Jiahn-Shing Lee } \\
\text { Taiwan }\end{array}$ & $\begin{array}{l}\text { Lyubomyr Lytvynchuk } \\
\text { Ukraine }\end{array}$ \\
\hline $\begin{array}{l}\text { Shingo Koyama } \\
\text { Japan }\end{array}$ & $\begin{array}{l}\text { Llewellyn Lee } \\
\text { Singapore }\end{array}$ & $\begin{array}{l}\text { Alex Macleod } \\
\text { UK }\end{array}$ \\
\hline $\begin{array}{l}\text { Igor Kozak } \\
\text { Saudi Arabia }\end{array}$ & $\begin{array}{l}\text { Hyung Lee } \\
\text { Korea, South }\end{array}$ & $\begin{array}{l}\text { Adriano Magli } \\
\text { Italy }\end{array}$ \\
\hline $\begin{array}{l}\text { Vassilios Kozobolis } \\
\text { Greece }\end{array}$ & $\begin{array}{l}\text { Christopher Seungkyu Lee } \\
\text { Korea, South }\end{array}$ & $\begin{array}{l}\text { George Magrath } \\
\text { USA }\end{array}$ \\
\hline $\begin{array}{l}\text { Florian Kretz } \\
\text { Germany }\end{array}$ & $\begin{array}{l}\text { Jong-Jer Lee } \\
\text { Taiwan }\end{array}$ & $\begin{array}{l}\text { Sajjad Mahmood } \\
\text { UK }\end{array}$ \\
\hline $\begin{array}{l}\text { Kaushal Kulkarni } \\
\text { USA }\end{array}$ & $\begin{array}{l}\text { Salomé Leibundgut } \\
\text { Switzerland }\end{array}$ & $\begin{array}{l}\text { Dimitra Makrynioti } \\
\text { Greece }\end{array}$ \\
\hline $\begin{array}{l}\text { Reetu Kundu } \\
\text { India }\end{array}$ & $\begin{array}{l}\text { Baihua Li } \\
\text { UK }\end{array}$ & $\begin{array}{l}\text { Baskaran Mani } \\
\text { Singapore }\end{array}$ \\
\hline $\begin{array}{l}\text { Kazuki Kuniyoshi } \\
\text { Japan }\end{array}$ & $\begin{array}{l}\text { Bin Li } \\
\text { China }\end{array}$ & $\begin{array}{l}\text { Gianluca Manni } \\
\text { Italy }\end{array}$ \\
\hline $\begin{array}{l}\text { Ajay Kuriyan } \\
\text { USA }\end{array}$ & $\begin{array}{l}\text { Qingfeng Liang } \\
\text { China }\end{array}$ & $\begin{array}{l}\text { Sonia Manning } \\
\text { Ireland }\end{array}$ \\
\hline $\begin{array}{l}\text { Ingela Lundin Kvalem } \\
\text { Norway }\end{array}$ & $\begin{array}{l}\text { Gerald Liew } \\
\text { Australia }\end{array}$ & $\begin{array}{l}\text { Ahmad Mansour } \\
\text { Lebanon }\end{array}$ \\
\hline $\begin{array}{l}\text { Hyung-Woo Kwak } \\
\text { Korea, South }\end{array}$ & $\begin{array}{l}\text { K. Sheng Lim } \\
\text { UK }\end{array}$ & $\begin{array}{l}\text { Kaweh Mansouri } \\
\text { Switzerland }\end{array}$ \\
\hline $\begin{array}{l}\text { Soon Il Kwon } \\
\text { Korea, South }\end{array}$ & $\begin{array}{l}\text { Faten Limaiem } \\
\text { Tunisia }\end{array}$ & $\begin{array}{l}\text { Srinivas Marmamula } \\
\text { India }\end{array}$ \\
\hline $\begin{array}{l}\text { Georgios Labiris } \\
\text { Greece }\end{array}$ & $\begin{array}{l}\text { Chun-Ju Lin } \\
\text { Taiwan }\end{array}$ & $\begin{array}{l}\text { Michael Marmor } \\
\text { USA }\end{array}$ \\
\hline $\begin{array}{l}\text { Francesco Lacarrubba } \\
\text { Italy }\end{array}$ & $\begin{array}{l}\text { Dusheng Lin } \\
\text { China }\end{array}$ & $\begin{array}{l}\text { Antonio Martinez } \\
\text { Spain }\end{array}$ \\
\hline $\begin{array}{l}\text { Neil Lagali } \\
\text { Sweden }\end{array}$ & $\begin{array}{l}\text { Paloma Liton } \\
\text { USA }\end{array}$ & $\begin{array}{l}\text { Enrico Martini } \\
\text { Italy }\end{array}$ \\
\hline $\begin{array}{l}\text { Jimmy Lai } \\
\text { Hong Kong }\end{array}$ & $\begin{array}{l}\text { Bingqian Liu } \\
\text { China }\end{array}$ & $\begin{array}{l}\text { Leonardo Mastropasqua } \\
\text { Italy }\end{array}$ \\
\hline $\begin{array}{l}\text { Klara Landau } \\
\text { Switzerland }\end{array}$ & $\begin{array}{l}\text { Stefan Löfgren } \\
\text { Sweden }\end{array}$ & $\begin{array}{l}\text { Suzana Matayoshi } \\
\text { Brazil }\end{array}$ \\
\hline $\begin{array}{l}\text { Alex Lau } \\
\text { Singapore }\end{array}$ & $\begin{array}{l}\text { Federico Luengo Gimeno } \\
\text { Argentina }\end{array}$ & $\begin{array}{l}\text { Sachin Mathew George } \\
\text { Singapore }\end{array}$ \\
\hline $\begin{array}{l}\text { Augustinus Laude } \\
\text { Singapore }\end{array}$ & $\begin{array}{l}\text { Fiona Luk } \\
\text { Hong Kong }\end{array}$ & $\begin{array}{l}\text { Hidetaka Matsumoto } \\
\text { Japan }\end{array}$ \\
\hline $\begin{array}{l}\text { Qihua Le } \\
\text { China }\end{array}$ & $\begin{array}{l}\text { Bruno Lumbroso } \\
\text { Italy }\end{array}$ & $\begin{array}{l}\text { Colm Mcalinden } \\
\text { UK }\end{array}$ \\
\hline
\end{tabular}




\begin{tabular}{|c|c|c|}
\hline Timothy Mcculley & Heather Moss & Els Ortibus \\
\hline USA & USA & Belgium \\
\hline $\begin{array}{l}\text { Martin Mckibbin } \\
\text { UK }\end{array}$ & $\begin{array}{l}\text { Marlene Moster } \\
\text { USA }\end{array}$ & $\begin{array}{l}\text { Nicola Orzalesi } \\
\text { Italy }\end{array}$ \\
\hline $\begin{array}{l}\text { Andrew Mcnaught } \\
\text { UK }\end{array}$ & $\begin{array}{l}\text { Eric Muir } \\
\text { USA }\end{array}$ & $\begin{array}{l}\text { Andrew Osborne } \\
\text { UK }\end{array}$ \\
\hline $\begin{array}{l}\text { Flavio Medina } \\
\text { Brazil }\end{array}$ & $\begin{array}{l}\text { Tomoaki Murakami } \\
\text { Japan }\end{array}$ & $\begin{array}{l}\text { Eoin O'Sullivan } \\
\text { UK }\end{array}$ \\
\hline $\begin{array}{l}\text { Carmen Mendez-Hernandez } \\
\text { Spain }\end{array}$ & $\begin{array}{l}\text { Ann Murchison } \\
\text { USA }\end{array}$ & $\begin{array}{l}\text { Atsushi Otani } \\
\text { Japan }\end{array}$ \\
\hline $\begin{array}{l}\text { Efstratios Mendrinos } \\
\text { Greece }\end{array}$ & $\begin{array}{l}\text { Ian Murdoch } \\
\text { UK }\end{array}$ & $\begin{array}{l}\text { Yoko Ozawa } \\
\text { Japan }\end{array}$ \\
\hline $\begin{array}{l}\text { Qianli Meng } \\
\text { China }\end{array}$ & $\begin{array}{l}\text { Jenny Myung } \\
\text { USA }\end{array}$ & $\begin{array}{l}\text { Joseph Panarelli } \\
\text { USA }\end{array}$ \\
\hline $\begin{array}{l}\text { Giuseppe Merla } \\
\text { Italy }\end{array}$ & $\begin{array}{l}\text { Kyung-Sun Na } \\
\text { Korea, South }\end{array}$ & $\begin{array}{l}\text { Georgios Panos } \\
\text { Switzerland }\end{array}$ \\
\hline $\begin{array}{l}\text { Carsten H. Meyer } \\
\text { Switzerland }\end{array}$ & $\begin{array}{l}\text { Zoltan Nagy } \\
\text { Hungary }\end{array}$ & $\begin{array}{l}\text { Eleni Papageorgiou } \\
\text { UK }\end{array}$ \\
\hline $\begin{array}{l}\text { Catherine Meyerle } \\
\text { USA }\end{array}$ & $\begin{array}{l}\text { Vinay Nangia } \\
\text { India }\end{array}$ & $\begin{array}{l}\text { Vasileios Papaliagkas } \\
\text { Greece }\end{array}$ \\
\hline $\begin{array}{l}\text { Zofia Michalewska } \\
\text { Poland }\end{array}$ & $\begin{array}{l}\text { Toshio Narimatsu } \\
\text { Japan }\end{array}$ & $\begin{array}{l}\text { Sang-Woo Park } \\
\text { Korea, South }\end{array}$ \\
\hline $\begin{array}{l}\text { Manuele Michelessi } \\
\text { Italy }\end{array}$ & $\begin{array}{l}\text { Piergiorgio Neri } \\
\text { Italy }\end{array}$ & $\begin{array}{l}\text { Ki Ho Park } \\
\text { Korea, South }\end{array}$ \\
\hline $\begin{array}{l}\text { Giovanni Milano } \\
\text { Italy }\end{array}$ & $\begin{array}{l}\text { Nuwan Niyadurupola } \\
\text { UK }\end{array}$ & $\begin{array}{l}\text { Hae-Young Park } \\
\text { Korea, South }\end{array}$ \\
\hline $\begin{array}{l}\text { Helen Mintz-Hittner } \\
\text { USA }\end{array}$ & $\begin{array}{l}\text { Hiroki Nomoto } \\
\text { Japan }\end{array}$ & $\begin{array}{l}\text { Annette Parkinson } \\
\text { UK }\end{array}$ \\
\hline $\begin{array}{l}\text { Ellen Mitchell } \\
\text { USA }\end{array}$ & $\begin{array}{l}\text { Miho Nozaki } \\
\text { Japan }\end{array}$ & $\begin{array}{l}\text { Mrinali Patel } \\
\text { USA }\end{array}$ \\
\hline $\begin{array}{l}\text { Masahiro Miura } \\
\text { Japan }\end{array}$ & $\begin{array}{l}\text { Francesco Oddone } \\
\text { Italy }\end{array}$ & $\begin{array}{l}\text { Niall Patton } \\
\text { UK }\end{array}$ \\
\hline $\begin{array}{l}\text { Sasan Moghimi } \\
\text { Iran }\end{array}$ & $\begin{array}{l}\text { Dominik Odrobina } \\
\text { Poland }\end{array}$ & $\begin{array}{l}\text { Mikk Pauklin } \\
\text { Estonia }\end{array}$ \\
\hline $\begin{array}{l}\text { Elad Moisseiev } \\
\text { Israel }\end{array}$ & $\begin{array}{l}\text { Akio Oishi } \\
\text { Japan }\end{array}$ & $\begin{array}{l}\text { Carlos Pavesio } \\
\text { UK }\end{array}$ \\
\hline $\begin{array}{l}\text { Jiten Morarji } \\
\text { UK }\end{array}$ & $\begin{array}{l}\text { Obiekwe Okoye } \\
\text { Nigeria }\end{array}$ & $\begin{array}{l}\text { Gökhan Pekel } \\
\text { Turkey }\end{array}$ \\
\hline $\begin{array}{l}\text { Kazuhiko Mori } \\
\text { Japan }\end{array}$ & $\begin{array}{l}\text { Refik Oltulu } \\
\text { Turkey }\end{array}$ & $\begin{array}{l}\text { Xijia Peng } \\
\text { China }\end{array}$ \\
\hline $\begin{array}{l}\text { Naoyuki Morishige } \\
\text { Japan }\end{array}$ & $\begin{array}{l}\text { Oluwole Omolase } \\
\text { Nigeria }\end{array}$ & $\begin{array}{l}\text { Henry Perry } \\
\text { USA }\end{array}$ \\
\hline $\begin{array}{l}\text { Paolo Morselli } \\
\text { Italy }\end{array}$ & $\begin{array}{l}\text { Cheung-Ter Ong } \\
\text { Taiwan }\end{array}$ & $\begin{array}{l}\text { George Peters } \\
\text { USA }\end{array}$ \\
\hline
\end{tabular}




\begin{tabular}{|c|c|c|}
\hline $\begin{array}{l}\text { Sergio Petroni } \\
\text { Italy }\end{array}$ & $\begin{array}{l}\text { Gabriella Ricciardelli } \\
\text { Italy }\end{array}$ & $\begin{array}{l}\text { Robert W Sault } \\
\text { Canada }\end{array}$ \\
\hline $\begin{array}{l}\text { Ioannis N. Petropoulos } \\
\text { UK }\end{array}$ & $\begin{array}{l}\text { Ivano Riva } \\
\text { Italy }\end{array}$ & $\begin{array}{l}\text { Judy Savige } \\
\text { Australia }\end{array}$ \\
\hline $\begin{array}{l}\text { Francesco Pichi } \\
\text { Italy }\end{array}$ & $\begin{array}{l}\text { Dana Robaei } \\
\text { Australia }\end{array}$ & $\begin{array}{l}\text { Giacomo Savini } \\
\text { Italy }\end{array}$ \\
\hline $\begin{array}{l}\text { Antonio Pinna } \\
\text { Italy }\end{array}$ & $\begin{array}{l}\text { Gloria Roberti } \\
\text { Italy }\end{array}$ & $\begin{array}{l}\text { Nicoline Schalij-Delfos } \\
\text { Netherlands }\end{array}$ \\
\hline $\begin{array}{l}\text { Susanne Pitz } \\
\text { Germany }\end{array}$ & $\begin{array}{l}\text { Alan Robin } \\
\text { USA }\end{array}$ & $\begin{array}{l}\text { Patrik Schatz } \\
\text { Sweden }\end{array}$ \\
\hline $\begin{array}{l}\text { Guillermo Plaza } \\
\text { Spain }\end{array}$ & $\begin{array}{l}\text { Murilo Roggia } \\
\text { Japan }\end{array}$ & $\begin{array}{l}\text { Victor Schepkin } \\
\text { USA }\end{array}$ \\
\hline $\begin{array}{l}\text { Pawan Prasher } \\
\text { India }\end{array}$ & $\begin{array}{l}\text { Peter Rolfe } \\
\text { Italy }\end{array}$ & $\begin{array}{l}\text { Katrina Schmid } \\
\text { Australia }\end{array}$ \\
\hline $\begin{array}{l}\text { Frank Proudlock } \\
\text { UK }\end{array}$ & $\begin{array}{l}\text { Teresa Rolle } \\
\text { Italy }\end{array}$ & $\begin{array}{l}\text { Dieter Schmidt } \\
\text { Germany }\end{array}$ \\
\hline $\begin{array}{l}\text { Malgorzata Przybylo } \\
\text { Poland }\end{array}$ & $\begin{array}{l}\text { Shisong Rong } \\
\text { China }\end{array}$ & $\begin{array}{l}\text { Lynn Schoenfield } \\
\text { USA }\end{array}$ \\
\hline $\begin{array}{l}\text { Renata Puertas } \\
\text { UK }\end{array}$ & $\begin{array}{l}\text { James Rosenbaum } \\
\text { USA }\end{array}$ & $\begin{array}{l}\text { Edina Schweighoffer } \\
\text { UK }\end{array}$ \\
\hline $\begin{array}{l}\text { Xuejiao Qin } \\
\text { China }\end{array}$ & $\begin{array}{l}\text { Luca Rossetti } \\
\text { Italy }\end{array}$ & $\begin{array}{l}\text { Michael Seider } \\
\text { USA }\end{array}$ \\
\hline $\begin{array}{l}\text { Guoping Qing } \\
\text { China }\end{array}$ & $\begin{array}{l}\text { Sylvain Roy } \\
\text { Switzerland }\end{array}$ & $\begin{array}{l}\text { Walter Sekundo } \\
\text { Germany }\end{array}$ \\
\hline $\begin{array}{l}\text { Yi Qu } \\
\text { China }\end{array}$ & $\begin{array}{l}\text { Eliana Rulli } \\
\text { Italy }\end{array}$ & $\begin{array}{l}\text { Jeremiah Seni } \\
\text { Tanzania }\end{array}$ \\
\hline $\begin{array}{l}\text { Luciano Quaranta } \\
\text { Italy }\end{array}$ & $\begin{array}{l}\text { Mohammad Sadiq } \\
\text { USA }\end{array}$ & $\begin{array}{l}\text { Parag Shah } \\
\text { India }\end{array}$ \\
\hline $\begin{array}{l}\text { Rajesh Rajagopalan } \\
\text { Singapore }\end{array}$ & $\begin{array}{l}\text { Osamah Saeedi } \\
\text { USA }\end{array}$ & $\begin{array}{l}\text { Syed Shah } \\
\text { USA }\end{array}$ \\
\hline $\begin{array}{l}\text { Raju Rajala } \\
\text { USA }\end{array}$ & $\begin{array}{l}\text { Wataru Saito } \\
\text { Japan }\end{array}$ & $\begin{array}{l}\text { Rupal Shah } \\
\text { India }\end{array}$ \\
\hline $\begin{array}{l}\text { Thiagarajan Raman } \\
\text { India }\end{array}$ & $\begin{array}{l}\text { Tsutomu Sakai } \\
\text { Japan }\end{array}$ & $\begin{array}{l}\text { Mahesh Shanmugam P } \\
\text { India }\end{array}$ \\
\hline $\begin{array}{l}\text { Manuel Ramirez } \\
\text { Mexico }\end{array}$ & $\begin{array}{l}\text { Daniel Salchow } \\
\text { Germany }\end{array}$ & $\begin{array}{l}\text { Shiwani Sharma } \\
\text { Australia }\end{array}$ \\
\hline $\begin{array}{l}\text { Pradeep Ramulu } \\
\text { USA }\end{array}$ & $\begin{array}{l}\text { Maria Letizia Salvetat } \\
\text { Italy }\end{array}$ & $\begin{array}{l}\text { Ismail Shatriah } \\
\text { Malaysia }\end{array}$ \\
\hline $\begin{array}{l}\text { Mohammad Reza Razeghinejad } \\
\text { Iran }\end{array}$ & $\begin{array}{l}\text { Roshini Sanders } \\
\text { UK }\end{array}$ & $\begin{array}{l}\text { Yasmin Shayesteh } \\
\text { USA }\end{array}$ \\
\hline $\begin{array}{l}\text { Andri Riau } \\
\text { Singapore }\end{array}$ & $\begin{array}{l}\text { Tatsuhiko Sato } \\
\text { USA }\end{array}$ & $\begin{array}{l}\text { Kei Shinoda } \\
\text { Japan }\end{array}$ \\
\hline $\begin{array}{l}\text { Joao Crispim Ribeiro } \\
\text { Brazil }\end{array}$ & $\begin{array}{l}\text { Simon Saule } \\
\text { France }\end{array}$ & $\begin{array}{l}\text { Chieko Shiragami } \\
\text { Japan }\end{array}$ \\
\hline
\end{tabular}


Stephen Shumack

Australia

Isabel Signes-Soler

Spain

Eduardo Silva

Chile

Huibert Jan Simonsz

Netherlands

Trefford Simpson

Canada

\section{Martin Sin}

Czech Republic

Michael Singer

USA

Rishi Singh

USA

Eric Singman

USA

Simon Skalicky

UK

Katarzyna Skonieczna

Poland

Frank Sloan

USA

Andrew Fraser Smith

Canada

Wendy M Smith

USA

Kamal Solaiman

Egypt

Peter Soliz

USA

Gianluca Sottilotta

Italy

Alex Spratt

UK

Oliver Stachs

Germany

Philip Stanley

Singapore

Johannes Steinberg

Germany

Douglas Stewart

USA
Irene Stratton

UK

Rupert Wolfgang Strauss

USA

Tobias Stupp

Germany

Tatiana Suarez

Spain

Ahalya Subramanian

UK

Prem Sagar Subramanian

USA

Young-Woo Suh

Korea, South

Rufino Suilva

Portugal

Michelle Sun

Australia

Hisaharu Suzuki

Japan

Takashi Suzuki

Japan

Meenakshi Swaminathan

India

Hiroki Takada

Japan

Johnson Tan

Singapore

Petrina Tan

Singapore

Suphi Taneri

Germany

Keiji Tanese

Japan

Shibo Tang

China

Masaki Tanito

Japan

Kristina Tarczy-Hornoch

USA

Simon Taylor

UK

Umit Y Tekelioglu

Turkey
Alejandro Tello

Colombia

Stephen Teoh

Singapore

Preethi Thiagarajan

USA

Margarita Todorova

Switzerland

Minoru Tomita

Japan

Paul Tomlins

UK

Marc Töteberg-Harms

Switzerland

Lisa Toto

Italy

Luis Trujillo

USA

Chieh-Chih Tsai

Taiwan

Rong Kung Tsai

Taiwan

Michael Tsatsos

UK

George Turner

UK

Addepalli Uday

India

Takashi Ueta

Japan

Marta Ugarte

UK

Canan Utine

Turkey

Monika Valtink

Germany

Suzanne Van Landingham

USA

Abhay Vasavada

India

Clemens Vass

Austria

Edbhergue Ventura Lola Costa

Brazil 


\author{
Nitin Verma \\ Australia \\ Monica Vetter \\ USA \\ Lingam Vijaya \\ India
}

Stephen Vincent

Australia

Francesco Viola

Italy

Gianni Virgili

Italy

Jan Albert Vos

Netherlands

Brendan Vote

Australia

Katrin Wacker

Germany

Nan-Kai Wang

Taiwan

Mengmeng Wang

China

Kai Jie Wang

China

Naushin Waseem

UK

Heather Waterman

UK

Alfred Wegener

Germany

Shihui Wei

China

Andreas Weinberger

Germany

Rong Wen

USA
Feng Wen

China

Adam Wenick

USA

Helmut Wilhelm

Germany

Mark Willcox

Australia

Geraint Williams

UK

Pete Williams

USA

Gabriel Willmann

Germany

Kate Windridge

UK

Yulia Wolfson

USA

Se Joon Woo

Korea, South

Emily Woodman

Australia

Wei-Chi Wu

Taiwan

Jason Yam

Hong Kong

Eylem Yaman Pinarci

Turkey

Morgan Yang

Singapore

Yan-Ning Yang

China

Paul Yang

USA

Yit Yang

UK
Tun K Yeo

Singapore

Gui-Shuang Ying

USA

Shao Onn Yong

Singapore

David Yoo

USA

Young Hee Yoon

Korea, South

Jing Yu

China

Shanshan Yu

China

Young Suk Yu

Korea, South

Tomasz Zarnowski

Poland

Hajo Zeeb

Germany

Xiongze Zhang

China

Sen Zhang

China

Zuoming Zhang

China

Yingfeng Zheng

China

Hua Zhong

China

Received: 15 January 2015 Accepted: 16 January 2015 Published: 30 January 2015

doi:10.1186/1471-2415-15-4

Cite this article as: Rice and Marshall: BMC

Ophthalmology reviewer acknowledgement

2014. BMC Ophthalmology 2015 15:4. 